\title{
Possible Long-Term Efficacy of Sitagliptin, a Dipeptidyl Peptidase-4 Inhibitor, for Slowly Progressive Type 1 Diabetes (SPIDDM) in the Stage of Non-Insulin-Dependency: An Open-Label Randomized Controlled Pilot Trial (SPAN-S)
}

\author{
Takuya Awata (D) Akira Shimada - Taro Maruyama • Yoichi Oikawa · Nobuyuki Yasukawa · Susumu Kurihara • \\ Yumi Miyashita · Masako Hatano · Yuichi Ikegami · Masafumi Matsuda • Masataka Niwa • Youichiro Kazama • \\ Shoichiro Tanaka · Tetsuro Kobayashi
}

Received: July 24, 2017 / Published online: September 19, 2017

(c) The Author(s) 2017. This article is an open access publication

\section{ABSTRACT}

Introduction: We tested the hypothesis that dipeptidyl peptidase-4 (DPP-4) inhibitors are effective in preserving the $\beta$-cell function for long-term periods in patients with slowly progressive type 1 diabetes (SPIDDM) or latent autoimmune diabetes in adults (LADA).

Taro Maruyama: deceased before study completion.

Enhanced content To view enhanced content for this article go to http://www.medengine.com/Redeem/ 18FBF0607C87BD83.

Electronic supplementary material The online version of this article (doi:10.1007/s13300-017-0299-7) contains supplementary material, which is available to authorized users.

T. Awata $(\bowtie) \cdot$ N. Yasukawa

Department of Diabetes, Endocrinology and

Metabolism, International University of Health and

Welfare Hospital, Iguchi, Nasushiobara-shi, Tochigi, Japan

e-mail: awatatakuya@gmail.com

A. Shimada · Y. Oikawa - S. Kurihara - M. Hatano ·

Y. Ikegami

Department of Endocrinology and Diabetes, Faculty of Medicine, Saitama Medical University, Saitama, Japan

T. Maruyama

Department of Internal Medicine, Saitama Social

Insurance Hospital, Saitama, Japan
Methods: In the present open-label, randomized, controlled trial, 14 non-insulin-requiring diabetic patients with glutamic acid decarboxylase autoantibodies (GADAb) were randomly assigned to receive either sitagliptin (S group) or pioglitazone (P group). As a historical control, the Tokyo Study, in which non-insulin-dependent patients with SPIDDM were assigned to receive treatment by either insulin or sulfonylurea (SU), was used.

Results: On average, the $\sum$ C-peptide values during the oral glucose tolerance test through the follow-up periods showed a nonsignificant increase in the $S$ group $(n=6, n=5$ at 48 months) compared to the $\mathrm{P}$ group $(n=5$, $n=2$ at 48 months). In comparison to the data in the Tokyo Study, treatment by sitagliptin significantly influenced the longitudinal

\section{Y. Miyashita}

Division of RI Laboratory, Biomedical Research

Center, Saitama Medical University, Saitama, Japan

\section{Matsuda}

Department of Endocrinology and Diabetes,

Saitama Medical Center, Saitama Medical

University, Saitama, Japan

M. Niwa

Iidabashi Medical Clinic, Tokyo, Japan

Y. Kazama

Kazama Medical Clinic, Yamanashi, Japan 
changes in the $\sum \mathrm{C}$-peptide values with a more increased direction than insulin or $\mathrm{SU}$, especially in patients with 48 months of follow-up ( $p=0.014$ and $p=0.007$, respectively). Although the titers of GADAb were not significantly different between the $\mathrm{S}$ and $\mathrm{P}$ groups during the study, the change ratio of the GADAb titers from baseline was significantly inversely correlated with the change ratio of the $\sum$ C-peptide values from baseline in the $\mathrm{S}$ group $(p=0.003)$; in particular, when the GADAb titers decreased from baseline, the $\sum \mathrm{C}$-peptide values frequently increased.

Conclusion: The present pilot trial suggests that treatment of SPIDDM/LADA by sitagliptin, a DPP-4 inhibitor, may be more effective in preserving the $\beta$-cell function than insulin treatment for at least 4 years, possibly through the immune modulatory effects of DPP-4 inhibitors.

Clinical trial registration: Japanese Clinical Trials Registry UMIN000003693.

Keywords: Dipeptidyl peptidase-4 (DPP-4) inhibitor; Intervention; Latent autoimmune diabetes in adults (LADA); Prevention; Sitagliptin; Slowly progressive type 1 diabetes (SPIDDM); Type 1 diabetes

\section{INTRODUCTION}

Type 1 diabetes (T1D) is caused by the autoimmune destruction of pancreatic $\beta$-cells, but it has been revealed that human T1D is substantially heterogeneous in its pathogenesis and natural history [1]. Slowly progressive insulin-dependent (type 1) diabetes mellitus (SPIDDM) [2, 3] or latent autoimmune diabetes in adults (LADA) [4-6] is characterized by the

S. Tanaka

Ai Home Clinic Toshima, Tokyo, Japan

T. Kobayashi

Division of Immunology and Molecular Medicine, Okinaka Memorial Institute for Medical Research,

Tokyo, Japan clinical phenotype of type 2 diabetes (T2D), such as adult onset, non-insulin independence at diagnosis, obesity and features of metabolic disorders, but has glutamic acid decarboxylase autoantibodies (GADAb) and/or islet cell antibodies (ICA); other antibodies such as insulinoma-associated antigen-2 autoantibodies (IA-2Ab) and insulin autoantibodies (IAA) are rather infrequent. Patients with SPIDDM/LADA frequently progress to an insulin-dependent state but with a slower rate of $\beta$-cell loss than in acute-onset T1D, probably because of the mild autoimmune attack. The prevalence of SPIDDM/LADA has been reported to range from $3 \%$ to $12 \%$ [7-10] of patients initially diagnosed with T2D in Western and other countries.

The generally unsatisfactory results of recent prevention/intervention trials for T1D have highlighted its heterogeneity $[11,12]$, and it has therefore been proposed that SPIDDM/LADA is an attractive target for immune intervention approaches [13, 14], given its milder autoimmune process and the longer preservation of the $\beta$-cell function than in acute-onset T1D. We previously conducted the Tokyo Study in Japanese patients with SPIDDM [15], which showed that, compared with sulfonylurea (SU), treatment with insulin slowed progression to an insulin-dependent state. The effect of early insulin therapy on SPIDDM/LADA has been confirmed [16].

Subsequent to the Tokyo study, we focused on dipeptidyl peptidase-4 (DPP-4) inhibitors as a promising treatment option for SPIDDM, since several studies have reported the beneficial effects of DPP-4 inhibitors in the NOD mouse, a mouse model of T1D [17-19]. We conducted the "study for the prevention of autoimmune non-insulin-dependent diabetes mellitus with sitagliptin" (SPAN-S) to evaluate the effects of sitagliptin, the first DPP-4 inhibitor released in Japan, on the glycemic control, preservation of $\beta$-cell function and longitudinal changes in islet cell antibodies. Although several studies in patients with SPIDDM/LADA have reported favorable results with DPP-4 inhibitors since the launch of this study [20-22], we continued our study for a longer period to assess the long-term efficacy of sitagliptin for SPIDDM/LADA. 


\section{METHODS}

\section{Patients}

This study was conducted in accordance with the International Conference on Harmonization Guidelines for Good Clinical Practice and the Declaration of Helsinki. The Saitama Medical University Ethics Committee approved this study, and the protocol was approved by the institutional review boards of the collaborating hospitals or the directors of the clinics. All of the procedures followed were in accordance with the ethical standards of the responsible committee on human experimentation (institutional and national) and with the Helsinki
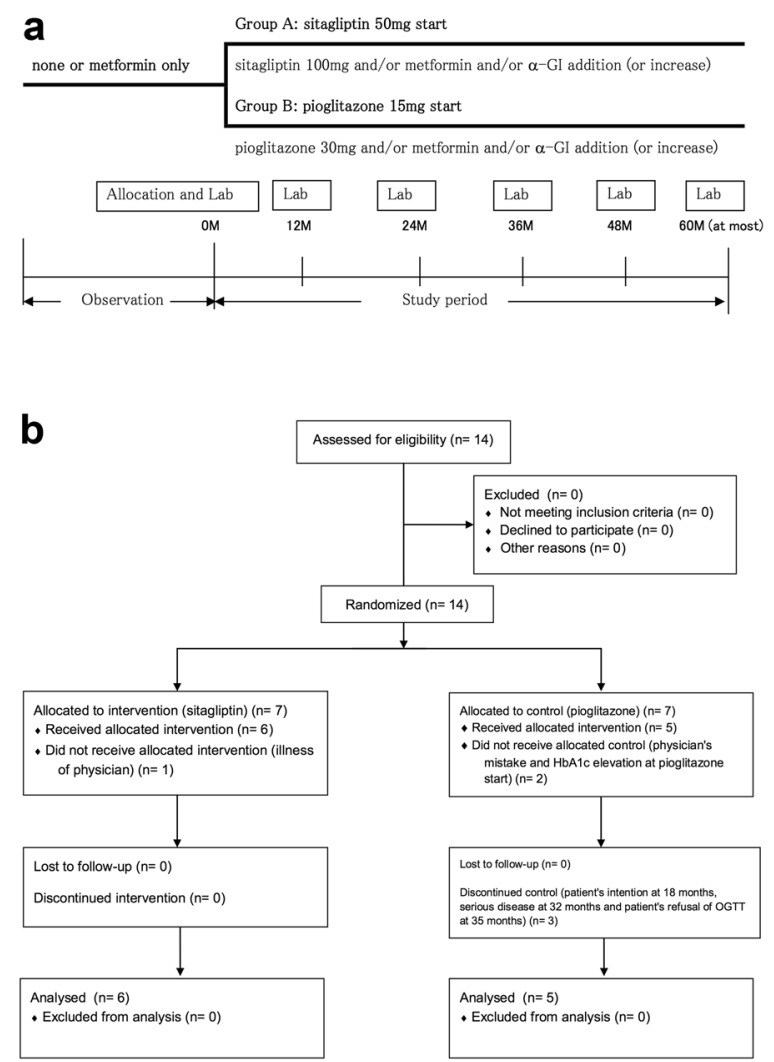

Fig. 1 Trial design and participant flow. a Schematic illustration of the trial design. $\mathbf{b}$ Participant flow. Data are presented as $n$. $\alpha$-GI alpha-glucosidase inhibitor, $L a b$ laboratory test, $M$ month, OGTT 75-g oral glucose tolerance test
Declaration of 1964, as revised in 2013. Written informed consent was obtained from all patients for being included in the study.

This was an open-label, prospective, randomized, controlled trial (Fig. 1). The protocol of this trial and supporting CONSORT checklist are available as Protocol S1 (original language), S2 (English translation) and Checklist S1. Participants were recruited from 10 June 2010, and follow-up data were collected until 23 February 2017. Fourteen SPIDDM patients were enrolled, and 11 of them were followed for at least 12 months (Fig. 1b). The inclusion criteria were as follows: (1) diabetes diagnosis and HbA1c level between $6.9 \%$ and $8.4 \%$, (2) GADAb-positive, (3) fasting serum C-peptide level $\geq 1.0 \mathrm{ng} /$ $\mathrm{ml}$, (4) age 20-79 years of age, (5) no treatment with oral glucose-lowering drugs except metformin within 2 months before enrollment and (6) no history of insulin or GLP-1 receptor antagonist treatment. The exclusion criteria were: (1) women who were pregnant or planning on becoming pregnant, (2) renal dysfunction with serum creatinine $\geq 1.5 \mathrm{mg} / \mathrm{dl}$ for males and $\geq 1.3 \mathrm{mg} / \mathrm{dl}$ for females, or creatinine clearance $\leq 50 \mathrm{ml} / \mathrm{min}$, and (3) patients who were deemed unsuitable for participation by their attending physician because of associated disease, complications or any other reasons. All subjects included in the study were Japanese.

The eligible patients, who were recruited from the collaborating hospitals or clinics, were all randomly assigned using a centralized internet web system to receive either sitagliptin or pioglitazone based on minimization adjusting for age, sex, HbA1c level, GADAb titer and metformin use before entry. Because the inferiority of SU compared to insulin treatment had been revealed in the Tokyo study, pioglitazone, a thiazolidinedione (TZD) approved in Japan, was selected as the comparator. The efficacy of TZD on SPIDDM/LADA has been evaluated in several studies with conflicting results [23, 24]. In addition, from an ethical point of view, patients under treatment with metformin were allowed to be enrolled because a possible favorable effect of metformin on SPIDDM/ LADA was suggested in our previous study [23]. 


\section{Study Design}

Patients were scheduled for visits at 2-month intervals for at least 12 months of follow-up. The dosage for the sitagliptin group was started at $50 \mathrm{mg}$ once per day and that for the pioglitazone group was $15 \mathrm{mg}$ once per day; if metformin was used at enrollment, the dosage was not changed. The target for glycemic control was set at an HbA1c level $<7.0 \%$. If glycemic control was not achieved during each visit, the regimens were intensified by either (1) increasing the dosage of sitagliptin or pioglitazone to 100 or $30 \mathrm{mg}$, respectively, and/or (2) adding metformin at $250-2250 \mathrm{mg}$ or increasing the dosage up to $2250 \mathrm{mg}$ and/or adding an alpha-glucosidase inhibitor (acarbose, voglibose or miglitol). Notably, SU agents were never used. The patients in both groups were switched to an insulin injection regimen when the HbA1c levels became $\geq 9.4 \%$ despite the above protocol of medication.

\section{Follow-up Assessment and Endpoint}

The patients' body weight, blood pressure, plasma glucose (PG) and HbA1c levels were measured every 2 months. All of the patients received an annual 75-g oral glucose tolerance test (OGTT) without receiving their morning dose of medication, and their islet antibodies (GADAb, IA-2Ab and IAA) were determined annually. The primary endpoints were the HbA1c values, the proportion of patients whose HbA1c values increased to $\geq 9.4 \%$, and the period from enrollment in which their HbA1c values increased to $\geq 9.4 \%$. The secondary endpoints included the sum of the serum C-peptide levels at $0,30,60,90$ and 120 min during the OGTT ( $\sum$ C-peptide), body mass index (BMI) and islet autoantibodies.

\section{Autoantibodies}

GADAb, IA-2Ab and IAA levels were determined in this study via radioimmunoassay (RSR Ltd., Cardiff, UK). With regard to the thyroid autoantibodies (TAb), thyroid peroxidase autoantibodies (TPOAb) and thyroglobulin autoantibodies
(TGAb) were determined by an electrochemiluminescence immunoassay (Roche Diagnostics $\mathrm{GmbH}$, Mannheim, Germany). Regarding the present GADAb assay, $10 \mathrm{U} / \mathrm{ml}$ corresponded to $180 \mathrm{U} / \mathrm{ml}$ (WHO units), the cutoff value that predicted further progression of $\beta$-cell dysfunction in the Tokyo study [25].

\section{Safety Assessments}

Any adverse events associated with the medications used in this study were evaluated and treated properly by physicians. Hypoglycemia was defined as a blood glucose level $<70 \mathrm{mg} / \mathrm{dl}$ $(3.9 \mathrm{mmol} / \mathrm{l})$, irrespective of hypoglycemic symptoms.

\section{Sample Size}

Based on our previous intervention trial (Tokyo Study) [15], in which 60 SPIDDM patients (30 each in the insulin and SU groups) were followed and the primary endpoint was the time at which the patient reached an insulin-dependent state (i.e., $\sum$ C-peptide $<4 \mathrm{ng} / \mathrm{ml}$ ), and on the more preserved $\beta$-cell function in the inclusion criteria of this trial, which would show deleterious progression of the function more clearly, we anticipated that a total of 40 patients (20 each in the $S$ and $P$ groups) would be a sufficient population in our initial plan for the SPAN-S. Although the primary endpoint in the present study was defined by HbA1c values, worsening of HbA1c values was expected to correspond well to worsening of $\sum$ C-peptide levels.

\section{Statistical Analysis}

Continuous data at baseline $(n=11)$ were first checked for their normality by the D'Agostino and Pearson test, revealing that the age, GADAb titer and change ratio of the GADAb titer from baseline significantly deviated from a normal distribution. Thus, the Mann-Whitney $U$ test and Wilcoxon signed rank test were applied to these data for comparisons between groups and comparison from the baseline within the same group, respectively. An unpaired $t$ test and 
paired $t$-test were used for duration, BMI, HbA1c, $\sum P G, \sum C$-peptide and change ratio of $\sum$ C-peptide from baseline, respectively. Categorical variables were compared using Fisher's exact test. To evaluate the difference in the longitudinal $\sum C$-peptide data or their change ratios between the treatment groups, the interaction between time and treatment assignment at entry was examined using a repeated measures analysis of variance (ANOVA). The correlation between the change ratio of the GADAb titers and that of the $\sum C$-peptide values was assessed using a nonparametric Spearman's correlation coefficient. Statistical significance was defined as $p<0.05$. The GraphPad Prism software program ver. 6 (GraphPad Software, Inc., La Jolla, CA, USA) was used for these tests.

\section{RESULTS}

\section{Patients and Follow-Up}

As shown in Fig. 1b, 14 patients were allocated: 7 were assigned sitagliptin (S group) and 7 pioglitazone (P group). Among them, six patients in the $S$ group and five patients in the $P$ group were followed for more than 1 year and analyzed.

The baseline characteristics of these 11 patients are shown in Table 1 . There were no significant differences between the two groups in age, sex, duration, metformin use before entry, BMI, HbA1c levels, GADAb titers or positivity of other autoantibodies (IA2Ab, IAA and $\mathrm{TAb})$.

Six and five patients in the $\mathrm{S}$ group were followed for 24 and 48 months, respectively, while five, four and two patients in the P group were followed for 12,24 and 48 months, respectively (Table S1). The intensification of the regimens after entry is shown in Table S2. Briefly, the sitagliptin dosage was not increased in any patients, and the pioglitazone dosage was increased in three patients (cases P2, P4 and P5). Metformin was added to two patients (case S5 and P5) among those who had not been treated by metformin at entry (cases S5, P1 and P5), and an alpha-glucosidase inhibitor (voglibose) was added to one patient (case S4). The
Table 1 Baseline characteristics of study patients with SPIDDM

\begin{tabular}{|c|c|c|c|}
\hline & $\begin{array}{l}\text { Sitagliptin } \\
\text { group } \\
(n=6)\end{array}$ & $\begin{array}{l}\text { Pioglitazone } \\
\text { group }(n=5)\end{array}$ & $p$ value \\
\hline Age (years) & $56 \pm 16$ & $57 \pm 8$ & 0.93 \\
\hline $\begin{array}{l}\text { Sex } \\
\qquad \text { (male:female) }\end{array}$ & $2: 4$ & $2: 3$ & 0.85 \\
\hline $\begin{array}{l}\text { Duration of } \\
\text { diabetes } \\
\text { (years) }\end{array}$ & $4.3 \pm 2.9$ & $3.8 \pm 4.1$ & 0.81 \\
\hline $\begin{array}{l}\text { Metformin use } \\
\text { before entry, } \\
n(\%)\end{array}$ & $5(83)$ & $3(60)$ & 0.48 \\
\hline $\mathrm{BMI}\left(\mathrm{kg} / \mathrm{m}^{2}\right)$ & $23.2 \pm 6.2$ & $23.9 \pm 3.9$ & 0.73 \\
\hline HbAlc (\%) & $7.5 \pm 0.5$ & $7.3 \pm 0.4$ & 0.59 \\
\hline GADAb (U/ml) & $\begin{array}{l}2.65 \\
\quad(1.6-250)\end{array}$ & $\begin{array}{l}8.4 \\
\quad(1.8-14,000)\end{array}$ & 0.93 \\
\hline $\begin{array}{l}\text { IA- } 2 \mathrm{Ab} \text { positive, } \\
n(\%)\end{array}$ & $2(33)$ & $1(20)$ & 0.70 \\
\hline $\begin{array}{l}\text { IAA positive, } \\
n(\%)\end{array}$ & $0(0)$ & $0(0)$ & na \\
\hline $\begin{array}{l}\text { TAb (TPOAb } \\
\text { and/or } \\
\text { TGAb) } \\
\text { positive, } n(\%)\end{array}$ & $2(33)$ & $3(60)$ & 0.46 \\
\hline
\end{tabular}

Data are $n(\%)$, mean \pm SD or median (range)

$B M I$ body mass index, GADAb glutamic acid decarboxylase autoantibodies, $I A-2 A b$ insulinoma-associated antigen-2 autoantibodies, $I A A$ insulin autoantibodies, $T A b$ thyroid autoantibodies, TPOAb thyroid peroxidase autoantibodies, $T G A b$ thyroglobulin autoantibodies, na not applicable

metformin dosage was changed in four patients (cases S2, S4, P3 and P4).

\section{Longitudinal Changes in the BMI, Blood Pressure and HbA1c}

The BMI (or body weight) of the $S$ group did not significantly differ from that at baseline; the BMI (or body weight) of the P group showed an 
increasing trend from the value at enrollment, but the change was not significant (Table S1). The blood pressure of both groups did not significantly differ from that at baseline (data not shown). The HbA1c levels were decreased in both groups, and significant reductions from baseline were observed at several points in the $S$ group: at 12 months in the patients with both 24 and 48 months of follow-up ( $n=6$ and $n=5$, respectively) and at 36 months in those with 48 months of follow-up (Table S1). The worst HbA1c levels in the S and P groups were $8.2 \%$ and $8.2 \%$, respectively (Table S2). The values of BMI (or body weight), blood pressure and HbA1c did not significantly differ between the two groups during the study.

\section{Longitudinal Changes in the C-peptide Response to the OGTT}

On average, the $\sum \mathrm{C}$-peptide values throughout the follow-up periods were greater in the $S$ group than in the P group, although a repeated-measure ANOVA showed that the influence of the treatment assignment on the longitudinal changes in the $\sum$ C-peptide values was not significant in any of the follow-up periods (Table S1). In the six patients in the $S$ group who were observed for at least 24 months, the $\sum C$-peptide values increased at 12 and 24 months (six patients) and decreased at 36 and 48 months (5 patients) but were still higher than those at baseline; furthermore, the increases at 12 months from baseline were significant $(p=0.049)$ in the patients with 12 and 24 months of follow-up (Table S1). In the five patients in the P group, of whom only two were followed more than 36 months, the $\sum \mathrm{C}$-peptide values were mostly unchanged from baseline (Table S1 and Table S2).

\section{Comparison of the $\beta$-Cell Function with Historical Control}

We compared our present data with those in the Tokyo Study as a historical control. In the Tokyo Study [15], the GADAb-positive non-insulin-requiring diabetic patients (SPIDDM), the same target subjects as in the present SPAN-S trial, were randomly assigned to receive either insulin
(I group) or SU (SU group) and were followed up for a maximum of 60 months. The longitudinal changes in the $\sum \mathrm{C}$-peptide values of the $\mathrm{S}$ and $\mathrm{P}$ groups were compared with those of the I and SU groups in the Tokyo Study. Of note, no significant differences were observed between the $S$ or $P$ group in the present study and the I or SU group in the Tokyo Study in the baseline characteristics of age, sex, duration, BMI, HbA1c levels, GADAb titers and $\sum C$-peptide values. Although the I group demonstrated better preservation of $\sum C$-peptide values than the SU group, as reported previously, the preservation of $\sum \mathrm{C}$-peptide values in the $S$ group in the present study appeared to be even better. As shown in Fig. 2a, a repeated-measures ANOVA revealed a significant interaction between time and treatment assignment (sitagliptin or insulin) as well as between time and treatment assignment (sitagliptin or $\mathrm{SU})$ in the patients with 48 months of follow-up ( $p=0.030$ and $p=0.00004$, respectively), indicating that treatment by sitagliptin significantly influenced the longitudinal changes of the C-peptide responses to the OGTT with a more increased direction than insulin and SU. Differences between the $\mathrm{S}$ group and the I and SU groups were further assessed based on the change ratios of the $\sum$ C-peptide values from baseline (Fig. 2b). The significant superiority of treatment by sitagliptin to that by insulin or SU in the preservation of the $\sum C$-peptide values appeared to be more evident in the patients with 48 months of follow-up $(p=0.014$ and $p=0.007$, respectively), although the significant superiority of sitagliptin to insulin or SU was also observed in the patients with 24 and 36 months of follow-up (data not shown). As for the P group, no significant superiority of pioglitazone compared to insulin or SU was observed in a repeated-measures ANOVA (data not shown).

\section{Individual Longitudinal $\sum$ C-peptide Values, GADAb Titers and IA2Ab Titers of the Patients in the $S$ Group with 48 Months of Follow-Up}

We were able to observe the clinical course for 48 months in 5 patients of the $S$ group (cases S1, S2, S3, S4 and S5 in Table S2). Individual 


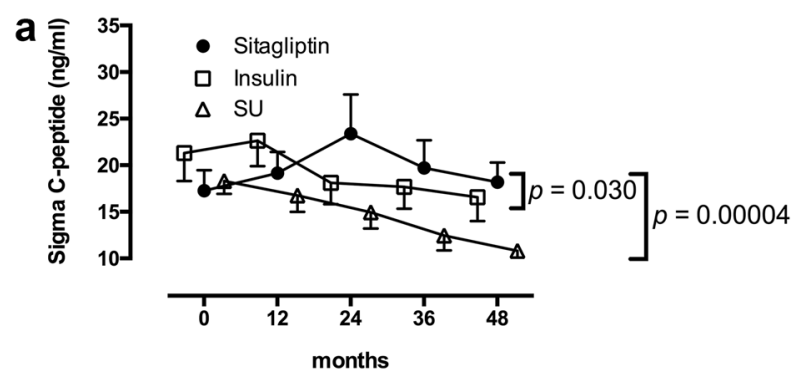

Fig. 2 Longitudinal changes in the C-peptide response to the OGTT for 48 months in the patients with sitagliptin treatment and in the Tokyo study. Patients with 48 months of follow-up are shown. The data are expressed as the mean \pm SEM. In both the $\sum \mathrm{C}$-peptide values (a) and change ratios from baseline (b), a

longitudinal change ratios of their $\sum \mathrm{C}$-peptide values, GADAb and IA-2Ab titers from baseline are shown in Fig. S1. Among these patients, four were heterozygous for the susceptible and neutral HLA haplotypes $(\mathrm{S} / \mathrm{N})$, and the other one (case S1) was homozygous for the neutral haplotype $(\mathrm{N} / \mathrm{N})$ [26]. Regarding TAb (TPOAb or TGAb), two patients (cases S3 and S5) were positive for TPOAb. The $\sum \mathrm{C}$-peptide values of all five patients were preserved during the 48 months of follow-up. Except for cases S2 and S5 whose $\sum$ C-peptide values remained almost the same throughout the study (S2) or slightly decreased from 24 to 48 months (S5), the values of the other three patients showed a tendency to increase from 12 to 24 months and then decreased from 36 to 48 months (Fig. S1). In comparison to cases S2, S3 and S5, who all had GADAb $>10 \mathrm{U} / \mathrm{ml}$ and two of whom (S2 and S3) were IA2Ab-positive, the $\sum$ C-peptide values of cases $\mathrm{S} 1$ and $\mathrm{S} 4$, who both showed $\mathrm{GADAb}<10 \mathrm{U} / \mathrm{ml}$ and were IA2Ab-negative, were increased to a greater degree from baseline, even at 48 months from entry. The relative increase from baseline was highest in case $\mathrm{S} 1$, who carried the HLA N/N genotype as well as both GADAb $<10 \mathrm{U} / \mathrm{ml}$ and IA2Ab-negative status.

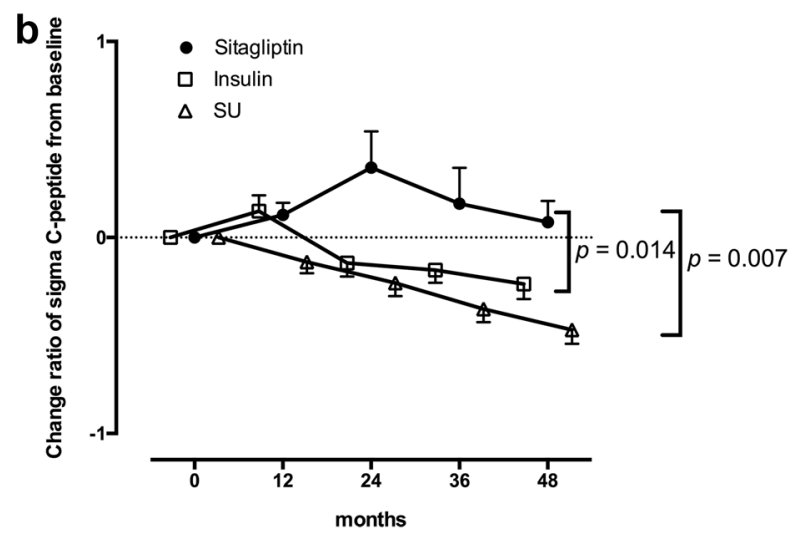

repeated-measures ANOVA revealed a significant interaction between time and treatment assignment (sitagliptin or insulin; $p=0.030$ and $p=0.014$, respectively) as well as between time and treatment assignment (sitagliptin or SU; $p=0.00004$ and $p=0.007$, respectively) in the longitudinal changes

\section{GADAb Titers}

The titers of GADAb were not significantly different between the $S$ and $P$ groups at any time point in the present study (Table S1). However, as shown in Fig. 3 , the change ratios of the GADAb titers from baseline were significantly inversely correlated with the change ratios of the $\sum \mathrm{C}$-peptide values from baseline in the $S$ group by a nonparametric analysis (Spearman's rank correlation coefficient $=-0.600, p=0.003$ ). In particular, when the change ratios of the GADAb titers from baseline were negative, those of the $\sum C$-peptide values were mostly positive, i.e., when the GADAb titers decreased from baseline, the $\sum \mathrm{C}$-peptide values frequently increased. In contrast, when the change ratios of the GADAb titers from baseline were positive, those of the $\sum \mathrm{C}$-peptide values tended to show values around zero (Fig. 3). In the P group, a nonsignificant inverse correlation was observed (Spearman's rank correlation coefficient $=-0.478$, $p=0.101$; Fig. S2).

\section{Hypoglycemia and Adverse Events}

No drug-related adverse events, including hypoglycemia, were observed. 


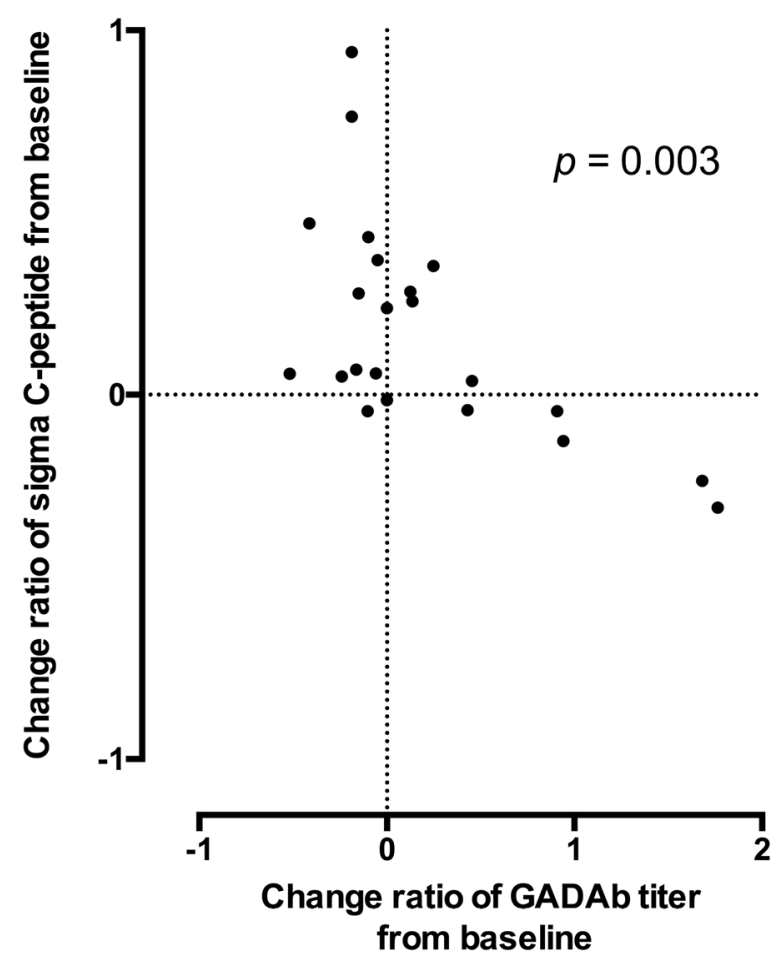

Fig. 3 The correlation between the change ratios of the GADAb titers and of the $\sum C$-peptide values from baseline in the $S$ group. Spearman correlation calculations revealed a significant inverse correlation between the change ratios of the GADAb titers and those of the $\sum C$-peptide values from baseline in the patients with sitagliptin treatment (Spearman's rank correlation coefficient $=-0.600, p=0.003$ )

\section{DISCUSSION}

In the present report, although the initial outcomes based on comparisons between sitagliptin and comparator (pioglitazone) were not evaluated enough-mostly because of the recruited sample size with low power-we presented our study as a pilot trial focusing on sitagliptin-treated patients with long-term (4 years) follow-up and further compared the effects of sitagliptin treatment on preserving the $\beta$-cell function with those of insulin or SU treatment in the patients enrolled in the Tokyo Study.

The present findings suggested that treatment by sitagliptin for SPIDDM/LADA is effective with respect to the preservation of the $\beta$-cell function as well as glycemic control. On average, the $\sum \mathrm{C}$-peptide values of the sitagliptin group were consistently higher than baseline for 48 months, with an apparent peak at 24 months. Compared to the data of the Tokyo Study as a historical control, in which SPIDDM patients were randomly assigned to treatment by either insulin or $\mathrm{SU}$, the preservation of the $\beta$-cell function following treatment by sitagliptin was significantly superior to that achieved with insulin and SU therapy, with the differences being evident from 24 months (Fig. 2b). We should be cautious of bias associated with using a historical control. Nevertheless, as described in the results section, no significant differences in the baseline characteristics were observed between the present study and the Tokyo Study. Furthermore, in the Tokyo Study, several patients showed a low $\beta$-cell function at baseline (defined as $\sum$ C-peptide $<10 \mathrm{ng} / \mathrm{ml}$ ), possibly because the enrolled patients in the Tokyo Study were recruited among those treated by SU, while none of the patients in the sitagliptin group of the present study showed such a low function. However, even when patients with a low $\beta$-cell function at baseline were excluded, the longitudinal changes in the $\sum$ C-peptide values in patients with 48 months of follow-up were significantly higher in the sitagliptin group than in the insulin and SU groups $(p=0.019$ and $p=0.014$ by repeated-measures ANOVA, respectively; data not shown).

The favorable effect of sitagliptin on the $\beta$-cell function may be because of the reduced glucotoxicity due to the improvement of glycemic control, as shown by the decline in the HbA1c values from baseline (Table S1). However, DPP-4 inhibitors may also have roles in the immune system. In the NOD mouse model of T1D, sitagliptin improved the rate of the islet graft survival partially by reducing the CD4 T cell numbers [19]. In another study, twice-daily treatment with NVP-DPP, a short-acting DPP-4 inhibitor, was able to reverse new-onset diabetes in NOD mice by increasing the number of $\mathrm{CD}^{+}{ }^{+} \mathrm{CD} 25^{+} \mathrm{FoxP}^{+}$regulatory $\mathrm{T}$ cells [18]. Recently, Alonso et al. reported that a DPP-4 inhibitor, MK626, prevented T1D in NOD mice in association with changes in the $\mathrm{CD}^{+} \mathrm{T}$ effector memory lymphocyte subset [27]. The 
superiority of sitagliptin to insulin in the preservation of the $\beta$-cell function might come from the immune regulatory effects of DPP-4 inhibitors on the autoimmune process.

Intriguingly, in the present study, we also observed that the change ratio of GADAb titers from baseline was significantly inversely correlated with that of $\sum C$-peptide in the $S$ group (Fig. 3). A higher GADAb titer in SPIDDM is a marker of both an activated $\mathrm{T}$ cell response to $\beta$-cell destruction and a high risk for progression to insulin dependence $[28,29]$. Therefore, the inverse correlation observed in the present study, especially the obvious association between the increase in the $\sum C$-peptide values from baseline and the decrease in the GADAb titers (Fig. 3), may reflect changes in the active autoimmune process of the patients influenced by the treatment with sitagliptin, which resulted in the alteration of the $\beta$-cell function in an inverse direction. As demonstrated in the individual longitudinal change ratios of their $\sum C$-peptide values from baseline (Fig. S1), the long-term preservation of the $\beta$-cell function ( $\sum$ C-peptide values) of patients in the $S$ group appeared to be associated with both GADAb $<10 \mathrm{U} / \mathrm{ml}$ and IA2Ab-negative status (cases S1 and S4) or the absence of the HLA class II susceptibility haplotype (case S1). However, further validation in a larger population of patients will be required to determine the immunologic and genetic factors influencing the efficacy of sitagliptin on the $\beta$-cell function.

Recently, based on the potential beneficial effects of DPP-4 inhibitors and the promising results in animal studies, DPP-4 inhibitors have been clinically tested in patients with T1D or SPIDDM/LADA. Favorable effects of sitagliptin were suggested in several cases with T1D [30] or SPIDDM/LADA [31, 32]; very recently, 4-year clinical remission of T1D in two young patients treated with sitagliptin and vitamin D3 were reported [33]. However, the results of clinical trials for T1D patients have been conflicting and appear to be inconclusive [34-36]. In contrast, the trials for patients with SPIDDM/LADA, in whom the effects of sitagliptin for 1 year [20], linagliptin for a maximum of 2 years [21] and saxagliptin for 6 months [22] have been evaluated, all reported favorable results concerning the preservation of the $\beta$-cell function, suggesting that DPP-4 inhibitors may be effective in SPIDDM/LADA patients as a class effect and that SPIDDM/LADA might be a good target in prevention/intervention trials. The present findings lend support to the previous reports on SPIDDM/LADA and further suggest that the beneficial immunologic effects of DPP-4 inhibitors may persist for a longer period in non-insulin-dependent patients with SPIDDM/ LADA than was observed in previous studies.

The major limitation of the present study is its small sample size, which substantially reduced the power of the study. The low number of enrolled patients may be due in part to the inclusion criteria of certain HbA1c values and no history of insulin treatment, since Japanese patients with SPIDDM under suboptimal glycemic control tend to be treated by insulin based on the results of the Tokyo Study. It should be noted that due to the small sample size, the present study lacks sufficient power to discriminate between the effects of sitagliptin and pioglitazone on SPIDDM and also insufficiently characterized the patients with SPIDDM/ LADA suitable for treatment with DPP-4 inhibitors. However, the observed efficacy of sitagliptin treatment on glycemic control and the preservation of the $\beta$-cell function appeared to be notable, particularly in the two patients who had GADAb $>10 \mathrm{U} / \mathrm{ml}$ and were IA2Ab-positive and heterozygous for susceptible and neutral HLA DRB1-DQB1 haplotypes [26] (Fig. S1B and S1C).

Another limitation is that metformin use was not prohibited in the present study from an ethical point of view. Metformin was used to treat five of the six patients in the $S$ group at baseline; during the study period, the dosage was increased in two patients and was added in one patient (Table S2). We previously reported that metformin may suppress the disease course of SPIDDM/LADA more effectively than pioglitazone [23], and we subsequently experienced a patient with SPIDDM/LADA whose $\beta$-cell function was maintained for 5 years with treatment of metformin alone [37]. Metformin increases the GLP-1 level [38], which regulates lymphocyte proliferation and the maintenance of peripheral regulatory $T$ cells [39]; given that 
metformin was recently reported to improve gut microbiome flora in type 2 diabetes patients [40], metformin treatment for non-insulin-dependent SPIDDM might have exerted immunomodulatory effects through gut flora modification. As such, metformin may be effective in preserving the $\beta$-cell function in addition to its glucose-lowering effects and thus the usage of metformin may have influenced the favorable results in the $S$ group. However, possible favorable effects of metformin on SPIDDM/LADA need to be verified in extensive clinical studies. Finally, although the use of an alpha-glucosidase inhibitor was also allowed in this trial, its influence would be minimal since only one patient received the medication and little is known of its role in SPIDDM/LADA.

\section{CONLUSION}

The present study suggested that treatment of SPIDDM/LADA by sitagliptin, possibly as a class effect of DPP-4 inhibitors, may be more effective in the preservation of the $\beta$-cell function than insulin treatment for at least 4 years, possibly through the immune modulatory effects of DPP-4 inhibitors. We therefore believe that this class of drugs may be an attractive choice in the treatment of SPIDDM/LADA, especially in the stage of non-insulin-dependency. Further large-scale and long-term studies are necessary to clarify the details of the beneficial effects of DPP-4 inhibitors in SPIDDM/LADA patients and possibly in acute-onset T1D patients with a residual $\beta$-cell function.

\section{ACKNOWLEDGEMENTS}

This work was funded via a grant from the Waksman Foundation of Japan, Inc. The funder had no role in any aspect of the study beyond funding. All authors made significant contributions to the study design, analysis and interpretation of data, as well as preparing and reviewing the manuscript. All named authors meet the International Committee of Medical Journal Editors (ICMJE) criteria for authorship for this manuscript, take responsibility for the integrity of the work as a whole and have given final approval for the version to be published. Previous presentation: some of the findings from this study were presented at the 58th Annual Meeting of The Japan Diabetes Society, Shimonoseki, Japan, 21-24 May 2015. The authors thank all of the members of the institutions joining the SPAN-S trial.

Disclosures. Takuya Awata, Akira Shimada, Taro Maruyama, Yoichi Oikawa, Nobuyuki Yasukawa, Susumu Kurihara, Yumi Miyashita, Masako Hatano, Yuichi Ikegami, Masafumi Matsuda, Masataka Niwa, Youichiro Kazama, Shoichiro Tanaka and Tetsuro Kobayashi have nothing to disclose.

Compliance with Ethics Guidelines. All procedures followed were in accordance with the ethical standards of the responsible committee on human experimentation (institutional and national) and with the Helsinki Declaration of 1964, as revised in 2013. Written informed consent was obtained from all patients for being included in the present study.

Data Availability. The data sets during the current study are available from the corresponding author on reasonable request.

Open Access. This article is distributed under the terms of the Creative Commons Attribution-NonCommercial 4.0 International License (http://creativecommons.org/licenses/ by-nc/4.0/), which permits any noncommercial use, distribution, and reproduction in any medium, provided you give appropriate credit to the original author(s) and the source, provide a link to the Creative Commons license, and indicate if changes were made.

\section{REFERENCES}

1. Atkinson MA, von Herrath M, Powers AC, Clare-Salzler M. Current concepts on the pathogenesis of type 1 diabetes-considerations for attempts to prevent and reverse the disease. Diabetes Care. 2015;38(6):979-88. 
2. Kobayashi T, Tamemoto K, Nakanishi $\mathrm{K}$, et al. Immunogenetic and clinical characterization of slowly progressive IDDM. Diabetes Care. 1993;16(5):780-8.

3. Tanaka S, Ohmori M, Awata T, et al. Diagnotic criteria for slowly progressive insulin-dependent (type 1) diabetes mellitus (SPIDDM) (2012): report by the comitteeon slowly progressive insulin-dependent (type 1) diabetes mellitus of the Japan Diabetes Society. Diabetol Int. 2015;6(1):1-7.

4. Tuomi T, Groop LC, Zimmet PZ, Rowley MJ, Knowles W, Mackay IR. Antibodies to glutamic acid decarboxylase reveal latent autoimmune diabetes mellitus in adults with a non-insulin-dependent onset of disease. Diabetes. 1993;42(2):359-62.

5. Pozzilli P, Di Mario U. Autoimmune diabetes not requiring insulin at diagnosis (latent autoimmune diabetes of the adult): definition, characterization, and potential prevention. Diabetes Care. 2001;24(8):1460-7.

6. Stenstrom G, Gottsater A, Bakhtadze E, Berger B, Sundkvist G. Latent autoimmune diabetes in adults: definition, prevalence, beta-cell function, and treatment. Diabetes. 2005;54(Suppl 2):S68-72.

7. Tanaka S, Awata T, Shimada A, et al. Clinical characteristics of slowly progressive insulin-dependent (type 1) diabetes mellitus (SPIDDM): 1st subcommittee report on SPIDDM, comittee on type 1 diabetes, Japan Diabetes Society. J Japan Diab Soc (in Japanese). 2011;54(1):65-75.

8. Tuomi T, Carlsson A, Li H, et al. Clinical and genetic characteristics of type 2 diabetes with and without GAD antibodies. Diabetes. 1999;48(1):150-7.

9. Zhou Z, Xiang Y, Ji L, et al. Frequency, immunogenetics, and clinical characteristics of latent autoimmune diabetes in China (LADA China study): a nationwide, multicenter, clinic-based cross-sectional study. Diabetes. 2013;62(2):543-50.

10. Maddaloni E, Lessan N, Al Tikriti A, Buzzetti R, Pozzilli P, Barakat MT. Latent autoimmune diabetes in adults in the United Arab Emirates: clinical features and factors related to insulin-requirement. PLoS ONE. 2015;10(8):e0131837.

11. Pozzilli P. Type 1 diabetes mellitus in 2011: heterogeneity of T1DM raises questions for therapy. Nat Rev Endocrinol. 2012;8(2):78-80.

12. Atkinson MA, Eisenbarth GS, Michels AW. Type 1 diabetes. Lancet. 2014;383(9911):69-82.

13. Kobayashi T, Nakanishi K, Murase T, Kosaka K. Small doses of subcutaneous insulin as a strategy for preventing slowly progressive beta-cell failure in islet cell antibody-positive patients with clinical features of NIDDM. Diabetes. 1996;45(5):622-6.

14. Pozzilli P, Maddaloni E, Buzzetti R. Combination immunotherapies for type 1 diabetes mellitus. Nat Rev Endocrinol. 2015;11(5):289-97.

15. Maruyama T, Tanaka S, Shimada A, et al. Insulin intervention in slowly progressive insulin-dependent (type 1) diabetes mellitus. J Clin Endocrinol Metab. 2008;93(6):2115-21.

16. Brophy S, Davies H, Mannan S, Brunt H, Williams $\mathrm{R}$. Interventions for latent autoimmune diabetes (LADA) in adults. Cochrane Database Syst Rev. 2011;9:CD006165.

17. Suarez-Pinzon WL, Cembrowski GS, Rabinovitch A. Combination therapy with a dipeptidyl peptidase- 4 inhibitor and a proton pump inhibitor restores normoglycaemia in non-obese diabetic mice. Diabetologia. 2009;52(8):1680-2.

18. Tian L, Gao J, Hao J, et al. Reversal of new-onset diabetes through modulating inflammation and stimulating beta-cell replication in nonobese diabetic mice by a dipeptidyl peptidase IV inhibitor. Endocrinology. 2010;151(7):3049-60.

19. Kim SJ, Nian C, McIntosh CH. Sitagliptin (MK0431) inhibition of dipeptidyl peptidase IV decreases nonobese diabetic mouse CD $4+\mathrm{T}$-cell migration through incretin-dependent and -independent pathways. Diabetes. 2010;59(7):1739-50.

20. Zhao Y, Yang L, Xiang Y, et al. Dipeptidyl peptidase 4 inhibitor sitagliptin maintains beta-cell function in patients with recent-onset latent autoimmune diabetes in adults: one year prospective study. J Clin Endocrinol Metab. 2014;99(5):E876-80.

21. Johansen OE, Boehm BO, Grill V, et al. C-peptide levels in latent autoimmune diabetes in adults treated with linagliptin versus glimepiride: exploratory results from a 2-year double-blind, randomized, controlled study. Diabetes Care. 2014;37(1):e11-2.

22. Buzzetti R, Pozzilli P, Frederich R, Iqbal N, Hirshberg B. Saxagliptin improves glycaemic control and C-peptide secretion in latent autoimmune diabetes in adults (LADA). Diabetes Metab Res Rev. 2016;32(3):289-96.

23. Shimada A, Shigihara T, Okubo Y, Katsuki T, Yamada Y, Oikawa Y. Pioglitazone may accelerate disease course of slowly progressive type 1 diabetes. Diabetes Metab Res Rev. 2011;27(8):951-3.

24. Zhou Z, Li X, Huang G, et al. Rosiglitazone combined with insulin preserves islet beta cell function in adult-onset latent autoimmune diabetes (LADA). Diabetes Metab Res Rev. 2005;21(2):203-8. 
25. Kobayashi T, Tanaka S, Shimada A, Maruyama T, et al. High titer of autoantibodies to GAD identifies a specific phenotype of adult-onset autoimmune diabetes: response to Buzzetti et al. Diabetes Care. 2007;30(11):e126.

26. Awata T, Kuzuya T, Matsuda A, Iwamoto Y, Kanazawa Y. Genetic analysis of HLA class II alleles and susceptibility to type 1 (insulin-dependent) diabetes mellitus in Japanese subjects. Diabetologia. 1992;35(5):419-24.

27. Alonso N, Julian MT, Carrascal J, et al. Type 1 diabetes prevention in NOD Mice by targeting DPPIV/ CD26 is associated with changes in CD8(+)T effector memory subset. PLoS ONE. 2015;10(11):e0142186.

28. Kasuga A, Maruyama T, Nakamoto S, Ozawa Y, Suzuki Y, Saruta T. High-titer autoantibodies against glutamic acid decarboxylase plus autoantibodies against insulin and IA-2 predicts insulin requirement in adult diabetic patients. J Autoimmun. 1999;12(2):131-5.

29. Shimada A, Maruyama T, Suzuki R, et al. Anti-GAD65 antibody titer may be important in assessing T-cell response in anti-GAD65 + diabetes with residual beta-cell function. Diabetes Care. 1999;22(10):1759.

30. Lima-Martinez MM, Guerra-Alcala E, Contreras M, Nastasi J, Noble JA, Polychronakos C. One year remission of type 1 diabetes mellitus in a patient treated with sitagliptin. Endocrinol Diabetes Metabol Case Rep. 2014;2014:140072.

31. Kandasamy N, Lennox G, Annamalai AK, Maguire G, Adler AI. Sitagliptin in glutamic acid decarboxylase antibody-positive diabetes mellitus. Endocr Pract. 2012;18(4):e65-8.

32. Rapti E, Karras S, Grammatiki M, et al. Combined treatment with sitagliptin and vitamin $\mathrm{D}$ in a patient with latent autoimmune diabetes in adults. Endocrinol Diabetes Metabol Case Rep. 2016;2016:150136.
33. Pinheiro MM, Pinheiro FM, Torres MA. Four-year clinical remission of type 1 diabetes mellitus in two patients treated with sitagliptin and vitamin D3. Endocrinol Diabetes Metabol Case Rep. 2016;2016:16-0099.

34. Ellis SL, Moser EG, Snell-Bergeon JK, Rodionova AS, Hazenfield RM, Garg SK. Effect of sitagliptin on glucose control in adult patients with Type 1 diabetes: a pilot, double-blind, randomized, crossover trial. Diabet Med. 2011;28(10):1176-81.

35. Garg SK, Moser EG, Bode BW, et al. Effect of sitagliptin on post-prandial glucagon and GLP-1 levels in patients with type 1 diabetes: investigator-initiated, double-blind, randomized, placebo-controlled trial. Endocr Pract. 2013;19(1):19-28.

36. Griffin KJ, Thompson PA, Gottschalk M, Kyllo JH, Rabinovitch A. Combination therapy with sitagliptin and lansoprazole in patients with recent-onset type 1 diabetes (REPAIR-T1D): 12-month results of a multicentre, randomised, placebo-controlled, phase 2 trial. Lancet Diabetes Endocrinol. 2014;2(9):710-8.

37. Hirata T, Shimada A, Morimoto J, Maruyama T. Slowly progressive type 1 diabetes treated with metformin for five years after onset. Intern Med. 2013;52(23):2635-7.

38. Bahne E, Hansen M, Bronden A, Sonne DP, Vilsboll $\mathrm{T}$, Knop FK. Involvement of glucagon-like peptide-1 in the glucose-lowering effect of metformin. Diabetes Obes Metab. 2016;18(10):955-61.

39. Hadjiyanni I, Siminovitch KA, Danska JS, Drucker DJ. Glucagon-like peptide-1 receptor signalling selectively regulates murine lymphocyte proliferation and maintenance of peripheral regulatory $\mathrm{T}$ cells. Diabetologia. 2010;53(4):730-40.

40. Forslund K, Hildebrand F, Nielsen T, et al. Disentangling type 2 diabetes and metformin treatment signatures in the human gut microbiota. Nature. 2015;528(7581):262-6. 